\title{
PRIMITIVE RINGS WITH INVOLUTION WHOSE SYMMETRIC ELEMENTS SATISFY A GENERALIZED POLYNOMIAL IDENTITY
}

WALLACE S. MARTINDALE III

Let $R$ be a primitive ring with involution *. Thus $R$ may be considered as an irreducible ring of endomorphisms of an additive abelian group $V$, so that $D=\operatorname{Hom}_{R}(V, V)$ is a division ring. Let $C$ be the center of $D$. We shall furthermore assume that $C R \subseteq R$. It can be shown that an involution $\gamma \rightarrow \bar{\gamma}$ is induced in $C$ which has the property that $\bar{\gamma} x=\left(\gamma x^{*}\right)^{*}$ for all $x \in R$. The involution ${ }^{*}$ is of the first kind if $\gamma \rightarrow \bar{\gamma}$ is the identity mapping and is of the second kind if there is a $\gamma \neq 0 \in C$ such that $\bar{\gamma}=-\gamma$. The set of symmetric elements of $R$ will be denoted by $S$.

We now assume that $S$ satisfies a nontrivial generalized polynomial identity over $C$ (in the sense of Amitsur). This means that there exists a nonzero element $f\left(x_{1}, x_{2}, \cdots, x_{n}\right)$ in the so-called $C$-universal product $R\langle x\rangle$ of the $C$-algebra $R$ and the free $C$-algebra $C\left[x_{1}, x_{2}, \cdots, x_{n}, \cdots\right]$ in noncommuting indeterminants $x_{1}, x_{2}$, $\cdots, x_{n}, \cdots$ such that $f\left(s_{1}, s_{2}, \cdots, s_{n}\right)=0$ for all $s_{1}, s_{2}, \cdots, s_{n} \in S$. For more precise details concerning the above notions we refer the reader to $[1, \S 4]$, and $[2, \S 3]$. The usual linearization process may be used so that we may assume without loss of generality that $S$ satisfies a nontrivial generalized (homogeneous) multilinear identity of degree $n$ in $x_{1}, x_{2}, \cdots, x_{n}$ :

$$
f=\sum \beta_{k} a_{i_{0}} x_{j_{1}} a_{i_{1}} \cdots a_{i_{n-1}} a_{j_{n}} a_{i_{n}}=0
$$

where each monomial is of the same fixed degree $n, \beta_{k} \in C$, and the $a_{k}$ 's are elements of $R$. Furthermore it is clear that one may assume that the $a_{k}$ 's belong to some fixed $C$-basis of $R$, and that for two distinct monomials in which the variables appear in the same order the corresponding sequences $\left(a_{i_{0}}, a_{i_{1}}, \cdots, a_{i_{n}}\right)$ and $\left(a_{i_{0}^{\prime}}, a_{i_{1}^{\prime}}, \cdots, a_{i_{n}^{\prime}}\right)$ of ring elements differ in at least one position.

Our object in this paper is to prove that, under the given conditions on our ring $R, D$ is finite dimensional over $C$ and $R$ contains nonzero transformations of finite rank. The proof rests heavily on an elementary but powerful lemma on vector spaces due to Amitsur [1, p. 211, Lemma 1], a specific version of which we state as follows:

Received by the editors June 26, 1969. 
LEMma 1 (AMITSUR). Let $V$ be a vector space over a field $F$ and let $b_{1}, b_{2}, \cdots, b_{m}$ be F-independent linear transformations of $V$. Then for any finite dimensional subspace $U_{0}$ of $V$ either there exists $v \in V$ such that $v b_{1}, v b_{2}, \cdots, v b_{m}$ are independent modulo $U_{0}$ or there is a nonzero transformation $b=\sum_{i=1}^{m} \alpha_{i} b_{i}, \alpha_{i} \in F$, of finite rank.

We are now ready to begin the study of the primitive ring $R$ with involution *, with $C R \subseteq R$, such that the symmetric elements $S$ satisfy a generalized multilinear identity of the form (1).

Without loss of generality we may assume that the involution ${ }^{*}$ is of the first kind. Indeed, if $\bar{\gamma}=-\gamma$ for some $\gamma \neq 0 \in C$, and $k \in K$, the set of skew elements of $R$, then $\gamma k \in S$ and consequently

$$
f\left(\gamma k, s_{2}, \cdots, s_{n}\right)=\gamma f\left(k, s_{2}, \cdots, s_{n}\right)=0
$$

for all $s_{2}, \cdots, s_{n} \in S$, thus forcing $f\left(k, s_{2}, \cdots, s_{n}\right)=0$. Repetition of this argument yields

$$
f\left(s_{1}+k_{1}, s_{2}+k_{2}, \cdots, s_{n}+k_{n}\right)=0
$$

for all $s_{i} \in S, k_{i} \in K$. Because the characteristic of $R$ is unequal to two, every element of $R$ is of the form $s+k, s \in S, k \in K$, and so $R$ itself satisfies the same generalized multilinear identity. By a theorem of Amitsur $[1$, p. 218, Theorem 10], $[D: C]<\infty$ and $R$ contains nonzero transformations of finite rank.

Let $F$ be a maximal subfield of $D$. Following Amitsur $[1$, p. 215, Lemma 5] we note that $R_{F}$, the subring of $\operatorname{Hom}(V, V)$ generated by $R$ and $F$, acts irreducibly on $V$ in the obvious way, with $\operatorname{Hom}_{R_{F}}(V, V)$ $=F$. Since the involution ${ }^{*}$ of $R$ is of the first kind it may be extended to an involution (again denoted by ${ }^{*}$ ) of $R_{F}$ according to the rule

$$
\left(\sum \alpha_{i} r_{i}\right)^{*}=\sum \alpha_{i} r_{i}^{*}, \quad \alpha_{i} \in F, \quad r_{i} \in R .
$$

$[1$, p. 215 , Lemma $6(\mathrm{~b})]$ insures that this mapping is well defined. Thus $F S$ is the set of symmetric elements of $R_{F}$ and satisfies the same generalized multilinear identity (1) as does $S$. Furthermore it is clear that (1) remains nontrivial over $F$.

At this point we claim that, in order to prove our main theorem, it suffices to show that $R_{F}$ contains a nonzero linear transformation of finite rank of $V$ over $F$. Indeed, this follows by $[1$, p. 216, Theorem 7]. Therefore, for the remainder of our proof, we are justified in assuming that to start with $D=C$ is a field. We suppose, for sake of argument, that $R$ does not contain a nonzero transformation of finite rank and aim at obtaining a contradiction. 
Lemma 2. Let $v_{1}, v_{2}, \cdots, v_{k}$ be $C$-independent vectors in $V$, let $b_{1}, b_{2}$, $\cdots, b_{m}$ be $C$-independent elements of $R$, and let $U_{0}$ be a finite dimensional subspace of $V$. Then there exists $s \in S$ such that $v_{1} s b_{1}, v_{1} s b_{2}, \cdots$, $v_{1} s b_{m}$ are independent modulo $U_{0}$ and $v_{i} s=0, i>1$.

Proof. First choose $x \in R$ such that $v_{1} x \neq 0$ and $v_{i} x=0, i>1$. Next note that $b_{1}^{*}, b_{2}{ }^{*}, \cdots, b_{m}^{*}$ are $C$-independent in $R$. By Lemma 1 , there exists $w \in V$ such that $w b_{1}^{*}, w b_{2}^{*}, \cdots, w b_{m}^{*}$ are independent modulo the subspace generated by $v_{1}, v_{2}, \cdots, v_{k}$. Thus one may pick $r \in R$ such that $v_{i} r=0, i=1,2, \cdots, k$ and $w b_{i}^{*} r=w b_{i}^{*}, i=1,2, \cdots, m$. If $\left\{b_{i}{ }^{*} r\right\}$ is a dependent set, then $\sum \lambda_{i}\left(b_{i}{ }^{*} r\right)=0$, some $\lambda_{i} \neq 0$. Consequently, $\sum \lambda_{i}\left(w b_{i}^{*} r\right)=\sum \lambda_{i}\left(w b_{i}^{*}\right)=0$, a contradiction to the independence of $\left\{w b_{i}^{*}\right\}$. Thus $\left\{b_{i}^{*} r\right\}$, and hence $\left\{r^{*} b_{i}\right\}$, is an independent set. Using Lemma 1 again, we can find $v \in V$ such that $\left\{v r^{*} b_{i}\right\}$ is independent modulo $U_{0}$. Now pick $t \in R$ so that $v_{1} x t=v$. Then $v_{1}\left(x t r^{*}+r t^{*} x^{*}\right) b_{j}=v r^{*} b_{j}, j=1,2, \cdots, m$ and $v_{i}\left(x t r^{*}+r t^{*} x^{*}\right)=0$ for $i=2,3, \cdots, k$. The proof of the lemma is complete, as we note $x t r^{*}+r t^{*} x^{*} \in S$.

Returning to the consideration of the generalized multilinear identity (1), we write (1) in the form $f=g+h$, where $g$ is the sum of all monomials in which the variables appear in the standard order (we may assume $g \neq 0$ ). We let $a_{01}, \cdots, a_{0 k_{0}}$ be the distinct (and hence independent) elements of $R$ which appear before $x_{1}$ in the monomials comprising $g$, and in general we let $a_{i 1} a_{i 2}, \cdots, a_{i k_{i}}$ denote those distinct elements among the $C$-basis $\left\{a_{i}\right\}$ which appear between $x_{i}$ and $x_{i+1}$ in all those monomials belonging to $g$ which start out in the form $a_{01} x_{1} a_{11} x_{2} \cdots a_{i-1,1} x_{i} \cdots$. We let $A$ denote the (necessarily finite dimensional) $C$-subspace of $R$ spanned by all the $a_{k}$ 's appearing in (1).

By Lemma 1 we can choose $v \in V$ such that $v a_{01}, v a_{02}, \cdots, v a_{0 k_{0}}$ are independent. Let $W_{0}=\sum_{j>1} C v a_{0 j}$, and let $U_{0}=v A$. By Lemma 2 we pick $s_{1} \in S$ such that $W_{0} s_{1}=0$ and $v a_{01} s_{1} a_{11}, \cdots, v a_{01} s_{1} a_{i k_{1}}$ are independent modulo $U_{0}$. Making repeated use of Lemma 2, we may choose a sequence

$$
W_{0}, U_{0}, s_{1}, W_{1}, U_{1}, s_{2}, \cdots, W_{n-1}, U_{n-1}, s_{n}
$$

as follows:

Let $W_{i}=\sum_{j>i} C v a_{01} s_{1} a_{11} s_{2} \cdots a_{i-1,1} s_{i} a_{i j}$.

Let $U_{i}=U_{0}+\sum v A s_{p_{1}} A s_{p_{2}} \cdots A s_{p_{l}} A$, where $p_{j} \leqq i$ and $l \leqq \imath$.

Choose $s_{i+1} \in S$ such that $\left\{v a_{01} s_{1} a_{11} \cdots s_{i} a_{i 1} s_{i+1} a_{i+1, j}\right\}$ is an independent set modulo $U_{i}$ and $W_{i} s_{i+1}=0$ and $U_{i-1} s_{i+1}=0$.

Now substitute $s_{1}, s_{2}, \cdots, s_{n}$ in the identity (1). We claim that 
any monomial in which the variables are permuted becomes 0 . Indeed, let $p$ be the first subscript not in the standard position, that is, the monomial has the form $b_{0} x_{1} b_{1} x_{2} \cdots x_{p-1} b_{p-1} x_{q} \cdots$, with $q>p$. Thus $v b_{0} s_{1} b_{1} s_{2} \cdots s_{p-1} b_{p-1} \in U_{p-1} \subseteq U_{q-2}$, and our claim is established since $U_{q-2} s_{q}=0$. Therefore $h\left(s_{1}, s_{2}, \cdots, s_{n}\right)=0$. On the other hand, by the way in which $s_{1}, s_{2}, \cdots, s_{n}$ were chosen, we finally see that

$$
0=f\left(s_{1}, s_{2}, \cdots, s_{n}\right)=\sum_{j} \lambda_{j} w_{j}
$$

where not all $\lambda_{j}=0$ and $w_{j}=a_{01} s_{1} a_{11} \cdots a_{n-1,1} s_{n} a_{n j}$. A contradiction results since $\left\{w_{j}\right\}$ is an independent set. This completes the proof of our main result, which we now state again.

THEOREM. Let $R$ be a dense ring of linear transformations of a vector space $V$ over a division ring $D$, with $C R \subseteq R$, where $C$ is the center of $D$. Furthermore, assume that $R$ has an involution and that the set $S$ of symmetric elements of $R$ satisfies a generalized polynomial identity over $C$. Then $D$ is finite dimensional over $C$ and $R$ contains nonzero transformations of finite rank.

Acknowledgment. We are indebted to the National Science Foundation, which partially supported the research involved in this paper.

\section{REFERENCES}

1. S. A. Amitsur, Generalized polynomial identities and pivotal monomials, Trans. Amer. Math. Soc. 114 (1965), 210-226. MR 30 \#3117.

2. W. S. Martindale III, Prime rings satisfying a generalized polynomial identity, J. Algebra 12 (1969), 576-584.

UNIVERSITY OF MASSACHUSETTS 